\title{
Make a Molecule: A Synthetic Organic and Medicinal Chemistry Workshop Program for High School Students
}

\author{
Iain A. Stepek ${ }^{1}$, Raphael Hofmann ${ }^{1}$, Paula L. Nichols ${ }^{1}$, Andrea Aschwanden ${ }^{2}$, Christophe Eckard ${ }^{2}$, Patrick \\ Aschwanden $^{2}$, and Jeffrey W. Bode ${ }^{1,3}$ *
}

${ }^{1}$ Laboratorium für Organische Chemie, Department of Chemistry and Applied Biosciences, ETH Zürich, 8093 Zürich, Switzerland

${ }^{3}$ Institute of Transformative Bio-Molecules (WPI-ITbM), Nagoya University, Chikusa, Nagoya 464-8602, Japan

\section{ABSTRACT}

Despite the importance of organic chemistry to human health and quality of life, very few outreach programs have been successful in communicating this message. This contributes to negative public perceptions of organic chemistry and obscures the enormous advances in quality of life engendered by organic synthesis. New chemical outreach programs that provide insight and hands-on experience of small molecule chemistry are vital to improving public engagement with the science. Make a Molecule is an organic chemistry workshop program that provides high school students with the opportunity to synthesize and biologically assess short peptides in a safe and easy manner, allowing them to experience the thrill of making and testing new bioactive molecules in a classroom environment. This manuscript details the development of the Make a Molecule project from its origins in our "synthetic fermentation" chemistry to its initial application in high schools. Scientific protocols were optimized to suit a classroom setting with the help of high school students. An accompanying website was constructed to serve as an experimental guide, aid with the workshop execution, and provide an educational resource for students and teachers. With everything in hand, the first Make a Molecule workshops were conducted in a local high school in Zurich, Switzerland, during which students were able to successfully synthesize and test novel bioactive molecules. 


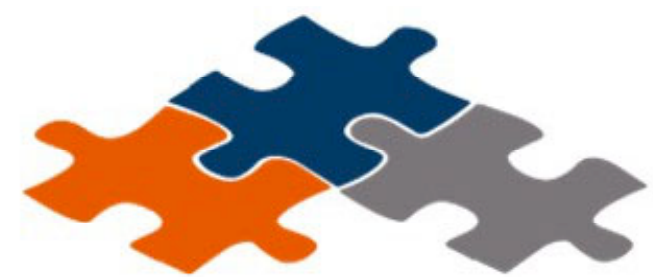

Make a Molecule

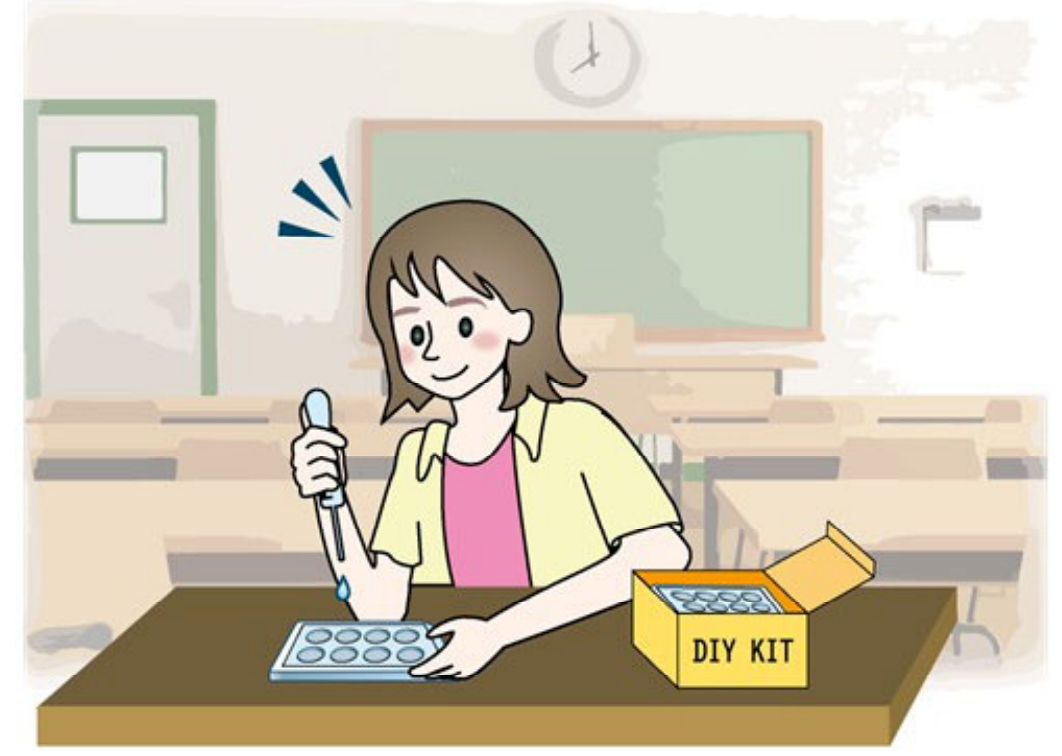

\section{KEYWORDS}

High school chemistry, organic chemistry, public outreach, hands-on learning, discovery learning, combinatorial chemistry, medicinal chemistry, microscale lab

\section{INTRODUCTION}

Organic Chemistry: Perception vs. Reality

Organic chemistry has played a pivotal role in many of the most beneficial scientific advances of the last century, including the discovery and development of antibiotics, medicines for the treatment of diseases, advances in new materials, and vast improvements for food production. These accomplishments, however, are not frequently associated with the field in the minds of the general public. A 2014 RSC survey on public perceptions of chemistry 
found that, while many people believed the field to be beneficial to society, many "lacked specific examples of how chemistry makes a positive impact, instead finding it easier to specify and visualize negatives or stereotypes." Perhaps due to this, the perception that organic chemistry (or even chemistry as a whole) is a dangerous and clandestine field, responsible for pollution, chemical waste and harmful substances, remains widespread among the public. ${ }^{2,3}$ Chemistry has also failed to engage the public emotionally, with more than $50 \%$ of people reporting "neutral" feelings for the science, leaving their views malleable and "vulnerable to stereotype." 1 In this environment, the popularity of TV shows like Breaking Bad - a television drama about illicit drug manufacturing that became perhaps the most prominent showcase of synthetic chemistry in popular culture for decades - becomes particularly damaging to the image of the science. The lack of opportunities for non-specialists to gain hands-on experience preparing new organic molecules reinforces these misconceptions and feelings of disconnection, raising a barrier between chemistry and the general public - to the detriment of both parties.

In part due to these perceived dangers of organic chemistry, very few outreach activities have been successful in effectively communicating how the synthetic chemistry is practiced and the value that it brings to modern life. In contrast, other disciplines such as computer science, ${ }^{4}$ robotics, ${ }^{5}$ astronomy, ${ }^{6}$ and even molecular biology ${ }^{7}$ have found great success in their efforts to involve schoolchildren and other non-specialists in their respective fields. These outreach efforts have contributed to the much greater public affection for these fields, despite the fact that chemistry arguably offers a much more direct impact on human health.

While much organic chemistry does indeed require specialist equipment and expertise, this is far from a universal truth. Many exciting and useful chemistries can be easily and safely performed on a simple high school bench-top, without the need for experimental experience or complicated scientific tools, and present very few potential hazards. Polymer chemists, with initiatives such as the University of Minnesota's "Polymer Day"8 and the partnership of UNC Wonder Connection with the Leibfarth group at the University of North Carolina ${ }^{9}$ have been utilising operationally simple and bench-safe reactions to showcase the application of organic chemistry in materials science. These programs have brought organic chemistry to high school students directly, sharing resources and strengthening educational networks. Analogous outreach programs for small molecule chemistry, however well-intentioned, have often focused more on demonstrations. ${ }^{10}$ In many cases, participants must travel to university laboratories to gain 
hands-on experiences, ${ }^{11}$ reinforcing the perception that organic molecules are dangerous and unsafe outside of the hands of experts in specialist environments.

It is our belief that through hands-on experience can public perceptions of chemistry as a difficult and dangerous science be altered. As such, there is a great need for the development of new outreach projects in small molecule organic chemistry, utilizing themes and experiments capable of providing a safe, informative and stimulating entry point into the discipline.

We recently introduced the concept of "synthetic fermentation" as a unique and powerful method for synthesizing complex organic molecules in water in a safe and predictable manner. ${ }^{12}$ These reactions occur spontaneously upon mixing the building blocks, without the need for added reagents, catalysts or organisms. As a result, the product mixtures obtained can be directly utilized in biological assays on plants, animals or cells without interference from toxic by-products (Figure 1).

The operational simplicity of synthetic fermentation was evident from the outset. In our first application of this concept, $\beta$-peptide libraries were generated by synthetic fermentation and assayed against HCV NS3/4A protease, leading to the identification of micromolar inhibitor 1 (Figure 1c). ${ }^{13} \mathrm{~A}$ library of 6000 small peptides was prepared from just 23 building blocks, simply by the combinatorial mixing of precursor stock solutions in a 96-well plate. The product mixtures generated were diluted and tested with a commercial assay kit without the need for prior purification. Active wells could be easily deconvoluted in focused screens to identify the active components and enable isolation of the inhibitor molecule. Each individual fermentation is performed on a sub-micromolar scale in aqueous media. An entire library of organic compounds can be prepared quickly from only a few milligrams of the building blocks. As such, the general safety concerns associated with organic chemistry are minimized. 
(a)

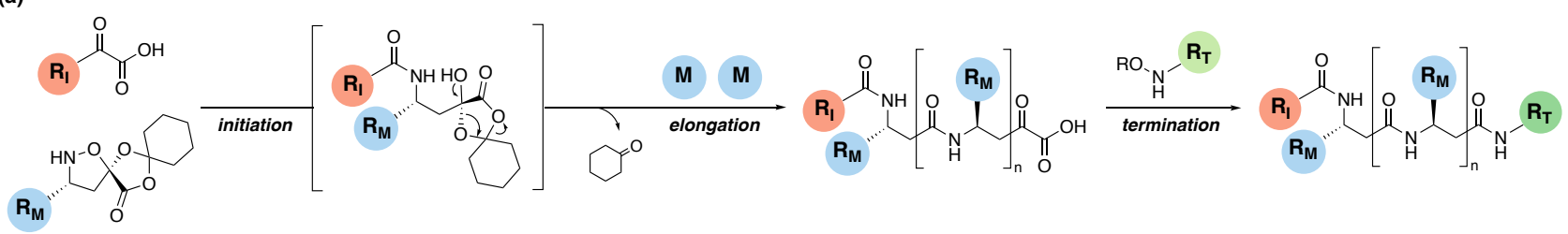

(b)
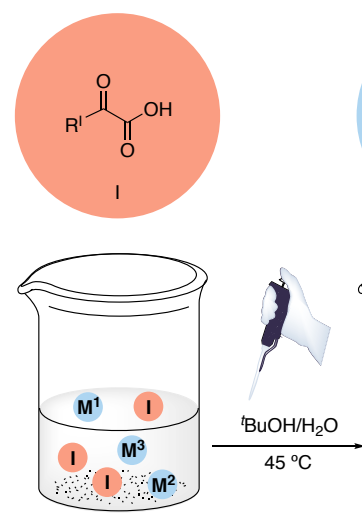

Initiation
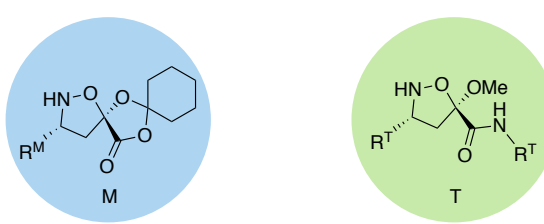

(c)

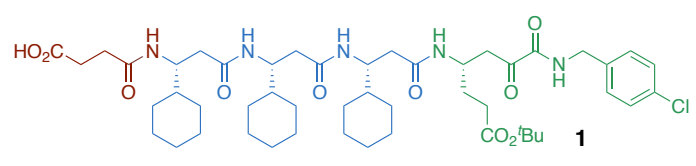

NS3/4A HCV protease inhibitor $\mathrm{IC}_{50}=1.0 \mu \mathrm{M}$

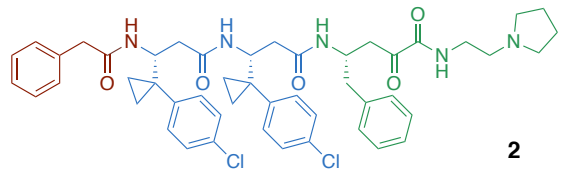

Antibacterial peptide MIC (Bacillus subtilis) $=5.7 \mu \mathrm{g} / \mathrm{mL}$

Examples of bioactive synthetic fermentation products

(d)

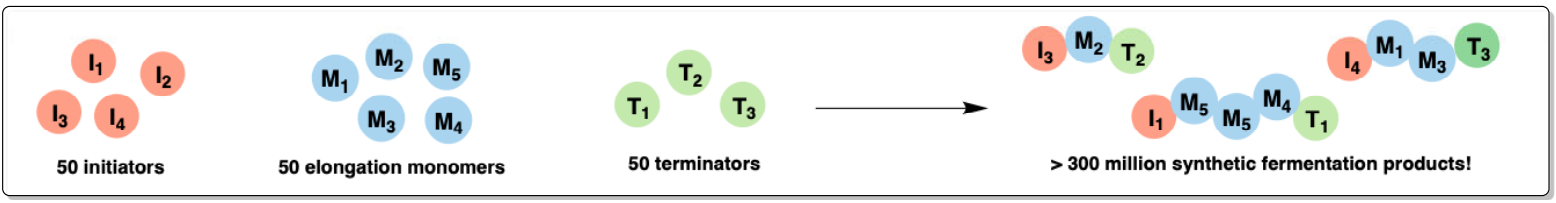

Figure 1. a) The chemical mechanism of the synthetic fermentation platform relies on the controlled KAHA ${ }^{14}$ oligomerization of elongation monomers $(\mathrm{M})$ upon reaction with initiators (I). The fermentation is stopped by addition of a terminator $(\mathrm{T})$. The resulting product mixtures can be directly screened in a biological assay after dilution, with no purification step required. (b) Generation of product mixtures with synthetic fermentation. (c) Examples of bioactive molecules identified and studied using the synthetic fermentation platform. (d) Large numbers of compounds can be quickly prepared from a small number of building blocks. ${ }^{12}$

Our story

After this early success in target-based screening, we aimed to test the capability of our synthetic fermentation platform towards phenotypic screening of chemical libraries for antibacterial activity. The gram-positive bacterium Bacillus subtilis was chosen as a target due to its straightforward handling, quick growth, and benign nature. Starting from an initially randomized building block screen, generating a potential library of around 8000 compounds, two active hit wells were identified. These wells were deconvoluted and subjected to focused screening, leading to the identification of an initial lead compound, which showed micromolar inhibition of bacterial growth. Further 
optimization and structure-activity relationship studies led to the discovery of peptide 2, which showed improved potency and selectivity for $B$. subtilis over human HEK293 cells (Figure 2). Subsequent proteomic studies indicated a mechanism of action involving interaction with penicillin binding proteins, similar to that of many $\beta$-lactam antibiotics. Binding affinity studies with penicillin binding protein 4 (PBP4) showed a nanomolar dissociation constant $\left(\mathrm{K}_{\mathrm{d}}\right)$, supporting our hypothesis. ${ }^{15}$

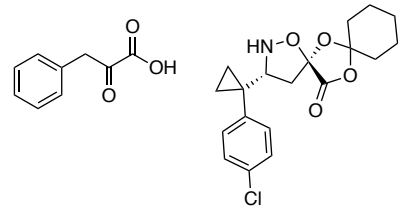

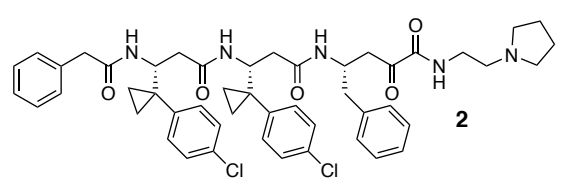

MIC (Bacillus subtilis): $5.7 \mu \mathrm{g} / \mathrm{mL}$

IC $_{50}$ (HEK293): > $100 \mu \mathrm{g} / \mathrm{mL}$

$K_{d}$ (PBP4): $364( \pm 19.1) n M$

Figure 2. Synthesis of optimized antibiotic 2 with synthetic fermentation. $\mathbf{2}$ showed improved activity in bacterial growth inhibition assays and reduced cytotoxicity relative to lead compounds. ${ }^{15}$

With these proof-of-concept findings, we obtained funding from the Swiss National Science Foundation AGORA program. The AGORA program aims to bridge the gap between research scientists and non-specialists, through the sponsorship of outreach projects that foster dialogue and allow research scientists and the general public the opportunity to understand and learn from one another. ${ }^{16}$ With this funding in place, we set out to develop a framework for the application of the synthetic fermentation platform in scientific outreach. With the help of preassembled synthetic fermentation "kits" and a guiding web app, we envisioned that the discovery of novel antibacterial molecules using synthetic fermentation could be an effective workshop tool for teaching high school students core concepts about organic synthesis, medicinal chemistry and the drug discovery process. By emphasizing the role of basic organic chemistry in the discovery and generation of bioactive molecules, and allowing the students to experience the excitement of making and testing novel compounds, we believe this approach presents an ideal platform to showcase organic chemistry as a force for good in society and to dismantle popular misconceptions surrounding the field.

\section{PROJECT DEVELOPMENT}

Optimization of Protocols 
Before the project could be brought to fruition, many aspects of the original research required adaptation to meet the needs and limitations of the desired high school workshop environment. In collaboration with Kantonsschule Zürich Nord, a local grammar school, three students conducted their Maturitätsarbeit (high school diploma) project in our laboratories. Each student was tasked with a separate project designed to probe the reproducibility of our data in the hands of high school students, as well as to optimize the protocols for an educational workshop setting.

The first project attempted to determine whether our previous results could be easily reproduced by high school students with no practical experience in organic chemistry. A student was provided a model building block set containing two initiators, four monomers and two terminators, including synthetic precursors to antimicrobial peptide 2. With only minimal guidance, the student designed a library set-up containing each permutation of the available building blocks, and tested each of the resulting reaction mixtures for bacterial growth inhibition against $B$. subtilis (Figure 3). After achieving comparable results to our initial test, the most active well was isolated, and the components subjected to a deconvolution study to identify which of the components are required for biological activity. In this way, the student was able to correctly identify the combination of building blocks $-\mathbf{I}^{\mathbf{1}}, \mathbf{M}^{\mathbf{4}}$ and $\mathbf{T}^{\mathbf{2}}-$ required for optimal activity in the bacterial assay. Additional tests carried out during this project that probed peptide length also provided valuable data for our understanding of the structure-activity relationships of the active molecule. ${ }^{17}$ 
(a)

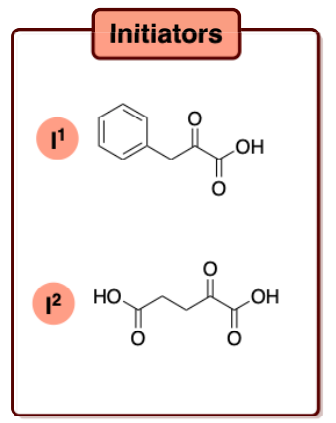

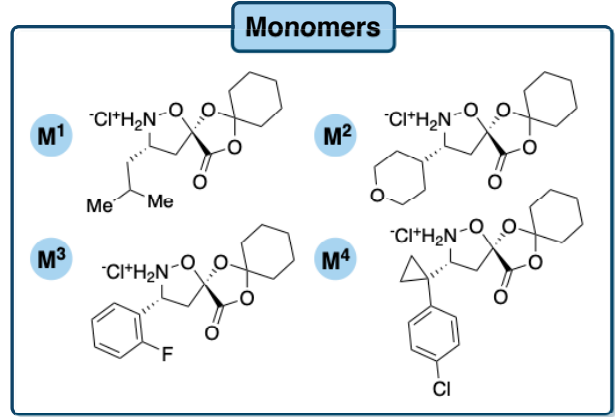

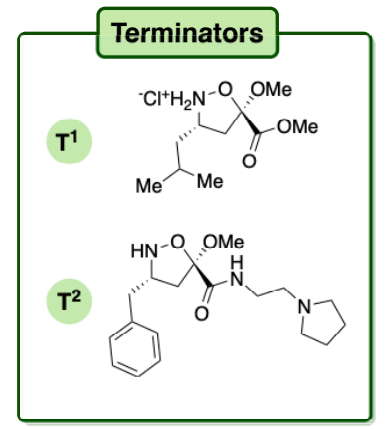

(b)

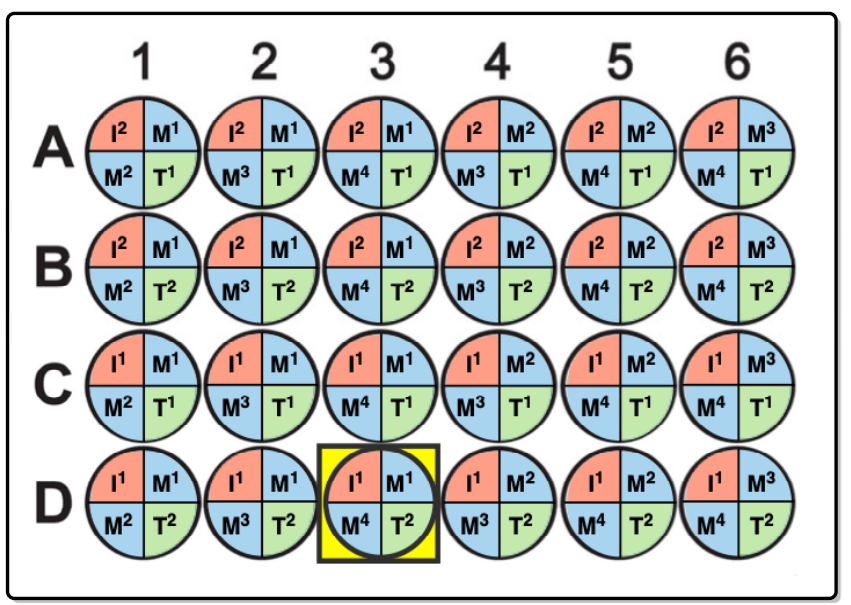

(c)

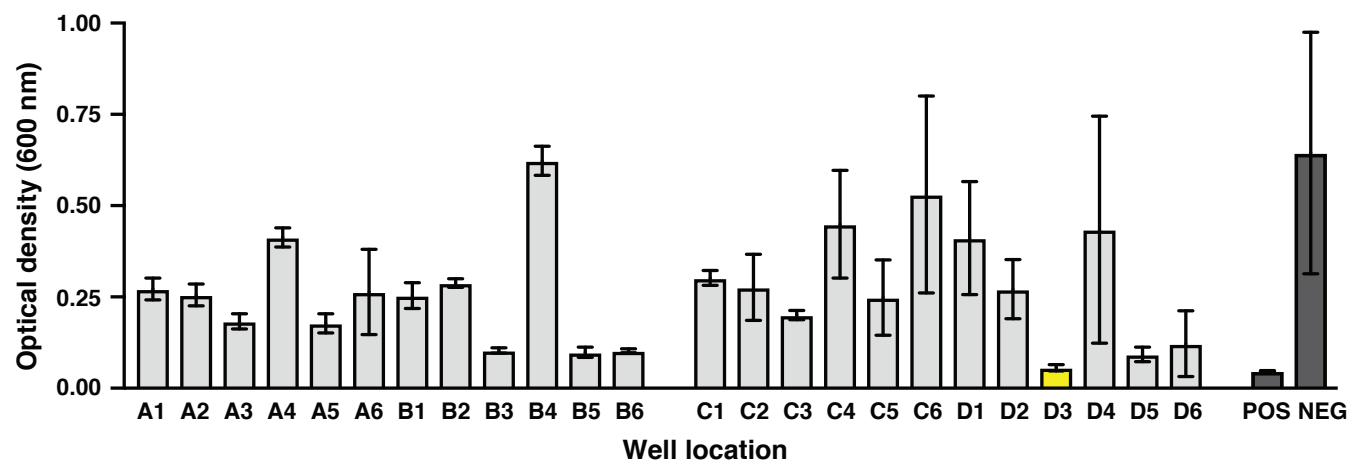

Figure 3. The preparation of small libraries and biological screening was carried out by two Maturitätsarbeit student. (a) The building block set provided, containing two initiators, four monomers and two terminators. (b) Well arrangement designed by the student for initial screening. Most active well is highlighted in yellow. (c) Results of the initial screen. Each well is charted against its optical density at $600 \mathrm{~nm}$ after incubation at $37^{\circ} \mathrm{C}$ for $24 \mathrm{~h}$. The most active well D3, containing precursors to active compound 2, highlighted in yellow. All values generated from average of three measurements. Positive and negative controls were provided by tetracycline $(150 \mu \mathrm{g} / \mathrm{mL}$ final concentration), or an equal volume of fermentation solvent ${ }^{t} \mathrm{BuOH} / \mathrm{H}_{2} \mathrm{O}(5: 1)$, respectively. ${ }^{17}$ 
(a)

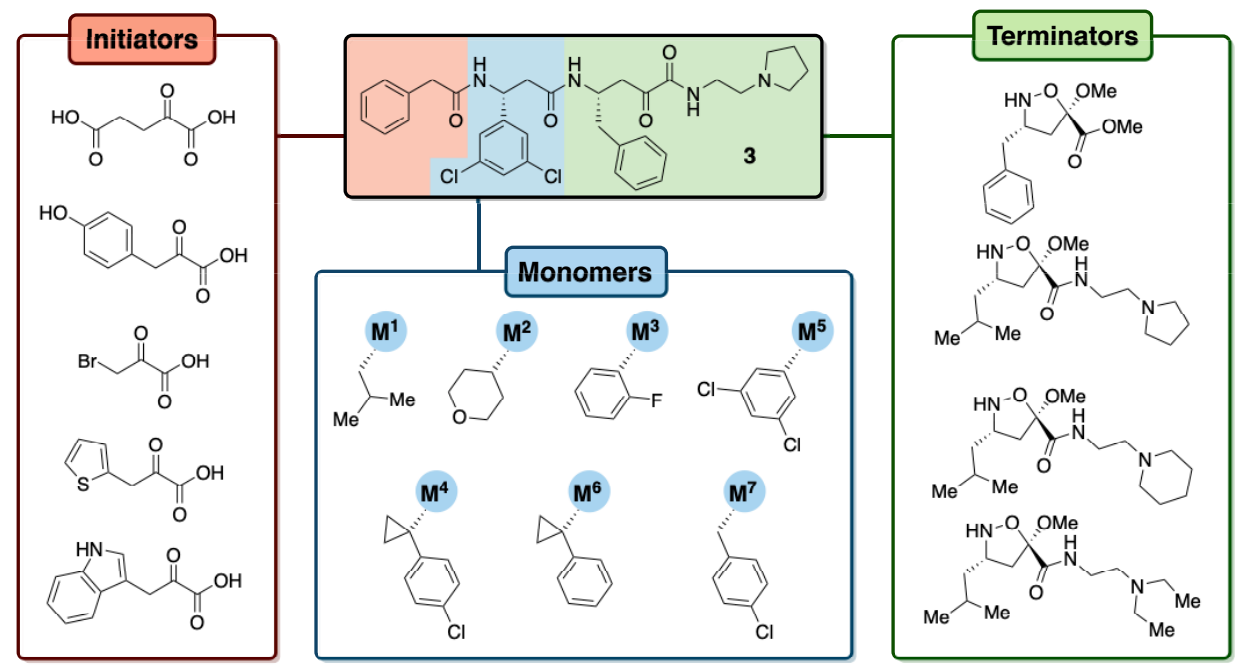

(b)

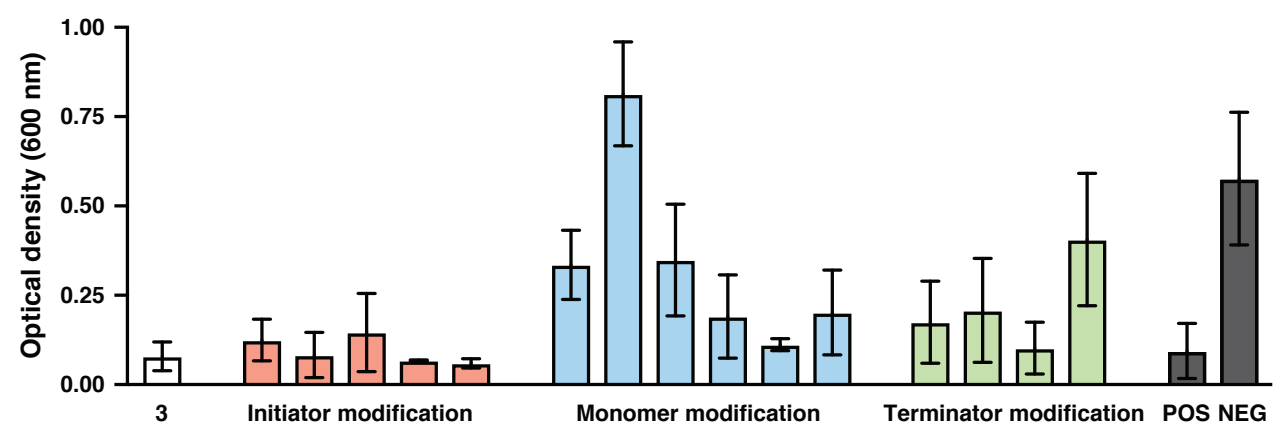

Figure 4. Structure-activity relationship studies carried out by the second Maturitätsarbeit student. (a) Variations to the initiator, monomer and terminator regions of lead molecule $\mathbf{3}$ were carried out. (b) The performance of these molecules in growth inhibition assay showed that initiator modification is tolerated, but a steep SAR profile exists for the monomer and terminator portions of the molecule, reproducing our previous findings. Positive and negative controls were provided by tetracycline $(150 \mu \mathrm{g} / \mathrm{mL}$ final concentration), or an equal volume of fermentation solvent ${ }^{t} \mathrm{BuOH} / \mathrm{H}_{2} \mathrm{O}(5: 1)$, respectively. ${ }^{18}$

A second student was provided with the structure of our active lead molecule $\mathbf{3}$, and tasked with varying each section of the molecule in turn. This project was intended to introduce medicinal chemistry concepts such as structure-activity relationships and pharmacophores to the students, as well as mimicking the lead optimization step of the drug discovery process. The student was able to find that, while modification of the initiator was tolerated, a steeper SAR curve was observed in the monomer and terminator regions, echoing our initial findings (Figure 4). ${ }^{18}$ Moreover, the optimized monomer was also successfully identified from a selection of available materials, indicating the reliability of our work setup, even in inexperienced hands. 
The third Maturitätsarbeit student was charged with optimising our assay protocol for application within a high school context. Our original assay, utilized in the other two projects (Figures 3 and 4), relied on an optical density measurement taken after 24 hours of bacterial incubation. This process was generally reliable and reproducible, but periodically generated false negative results due to compounds precipitating from solution and coating the base of the 96-well plate. Moreover, this assay required the use of a plate reader for assay readout, and lacked the visual qualities that we considered ideal for a school environment. We also hoped to reduce the assay time so as to more easily fit the synthetic fermentation protocol into a two-day workshop period.

While initial attempts at agar plate-based assay methods failed to show promise, success was found in the adaptation of the optical readout to a colorimetric method by the addition of resazurin dye. Resazurin undergoes a reduction-induced color change from blue (resazurin) to pink (resorufin) in the presence of living cells, so the antibacterial properties of each fermentation mixture can be assessed simply by viewing the color of the wells (Figure 5a). We were particularly pleased to observe that the optimal assay duration for maximal colorimetric contrast with this approach proved to be $2-4$ hours, a timeframe perfectly suited for a school day workshop (Figure $5 b)$.

(a)

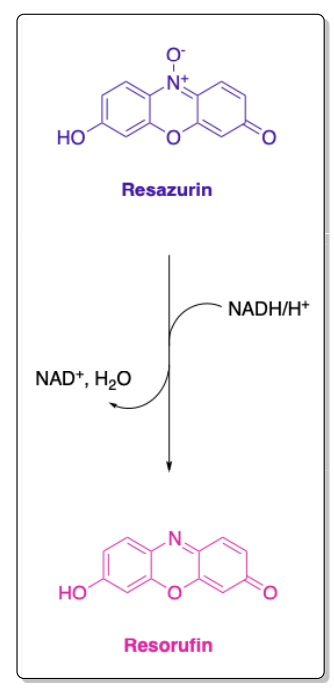

(b)

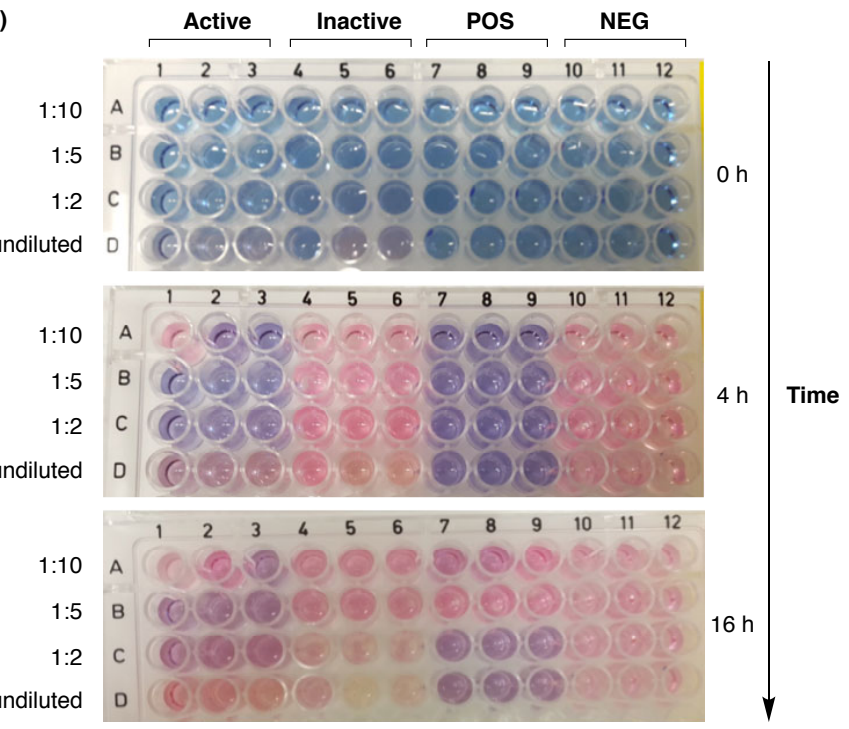

Figure 5. The resazurin-mediated colorimetric assay optimized for use in the project. (a) Resazurin is reduced to resorufin in the presence of living cells, causing a color change from blue to pink. (b) Assay progression for active and inactive synthetic fermentation mixtures (alongside positive and negative controls) is shown, over a period of sixteen hours. Twofold dilution of the synthetic fermentation cultures with PBS prior to the assay, and an assay time 
of 2-4 hours was shown to be optimal. Positive and negative controls were provided by tetracycline $(150 \mu \mathrm{g} / \mathrm{mL}$ final concentration), or an equal volume of fermentation solvent ${ }^{t} \mathrm{BuOH} / \mathrm{H}_{2} \mathrm{O}(5: 1)$, respectively. ${ }^{19}$

This project also allowed us to optimize other aspects of the new assay protocol. We observed in initial tests that the color of the resazurin was sensitive to $\mathrm{pH}$, and the synthetic fermentation reaction mixtures are acidic in nature due to the hydrochloride salt building blocks used. As a result, the addition of the reaction mixtures directly into the assay resulted in a faded color spectrum. However, neutralization of the fermentation cultures through twofold dilution with PBS buffer prior to the assay was sufficient to overcome this problem (Figure $5 b$ ). These modifications to the assay protocol were adopted as a standard procedure for the Make a Molecule workshops. ${ }^{19}$

With our optimized protocols in hand, we turned to the design of the project's accompanying website and web application. We envisioned the construction of a web app that would not only provide practical tips and guide the students through the experimental process, but also act as a database to compile the results, uncover trends in the data, and identify new, previously untested bioactive molecules synthesized by participating students. To achieve this, we contracted a web design firm to develop a full version of this application (https://makeamolecule.org/building_blocks; log in required). This program allows the user to design their experiments by dragging and dropping building blocks into their desired wells (Figure 6). After this input is complete, a synthetic fermentation protocol based on their experimental design can be automatically generated for the student to print and use. After the experiment has been conducted, the student can dictate their experimental observations using the "report results" function. This data is collected in the application, allowing for the collation of a vast database of biological assay results acquired from the student participants. To provide further data, the students can also attach a photo of their assay plates to their reported results (Figure 7). 


\section{hy Building Blocks}

Choose your block, next drag and dropped into wells. To show structure and properties, click over building block.

$\frac{1 \text { Initiators }}{11} 1213$

Monomers

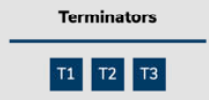

Figure 6. Interface of the web app showing the experimental design function. Building blocks can be placed into reaction wells of choice by a drag-and-drop mechanism, and a reaction protocol for each student is generated based on the well design chosen.
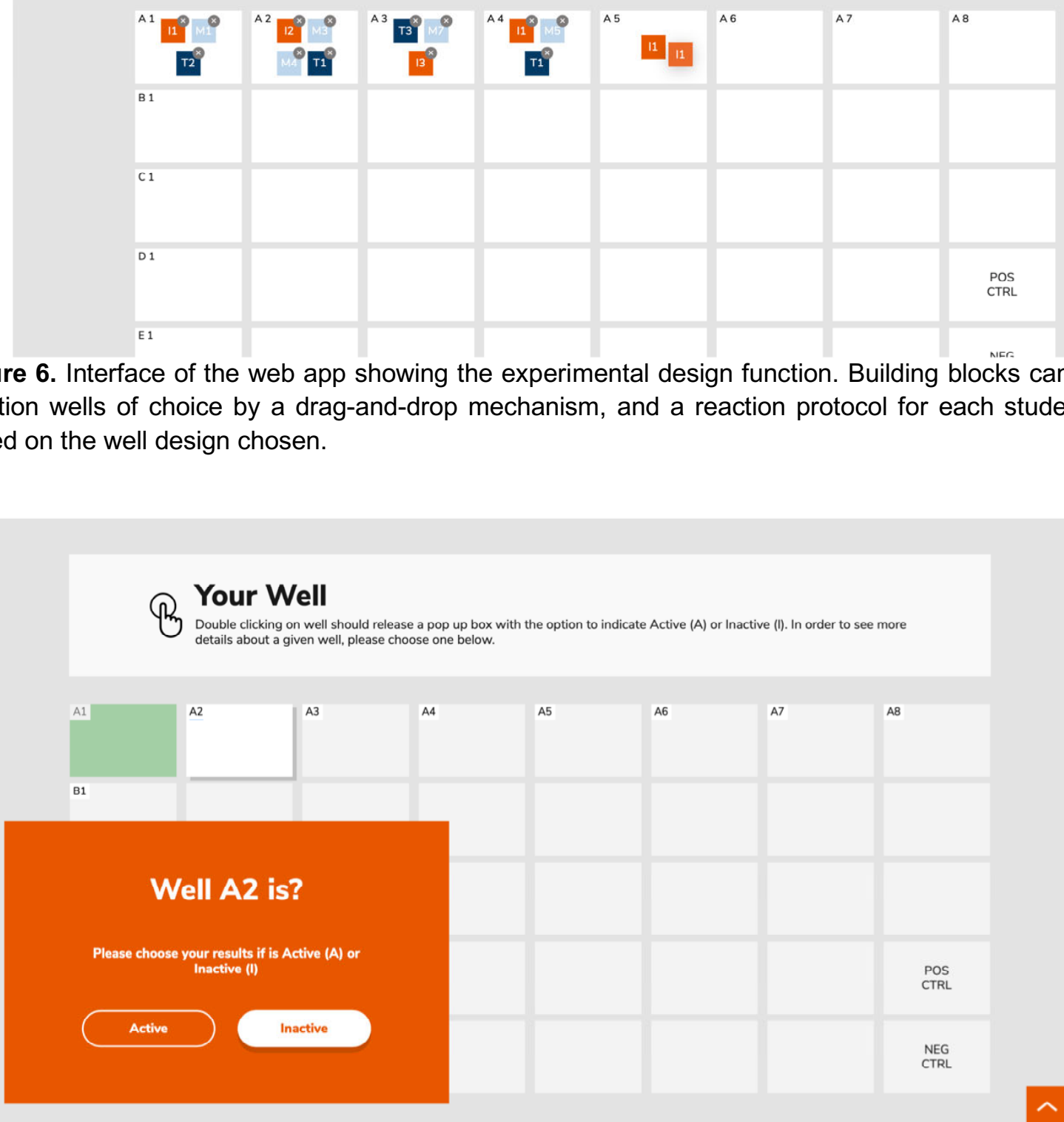

Figure 7. The "report results" function in the web app allows students to describe their scientific observations and upload photos of their assay results, which are stored as part of a larger database inside the application.

To complement the web app on our webpage, we aimed to build an educational resource for both chemistry students and teachers. Our Make a Molecule website now hosts a series of practical tips videos to aid the students with their 
experiments and an "Ask a Scientist" forum feature, allowing students to ask questions or seek assistance from experts in the field about the Make a Molecule program or related areas. We also sought to contextualize the experimental side of the workshop by providing background information on antibiotics, drug discovery and organic chemistry as a whole. A careers section for nascent young chemists was also included, featuring testimonials from chemists in varied industries about their diverging professional paths and their advice for students interested in pursuing chemistry (Figure 8).

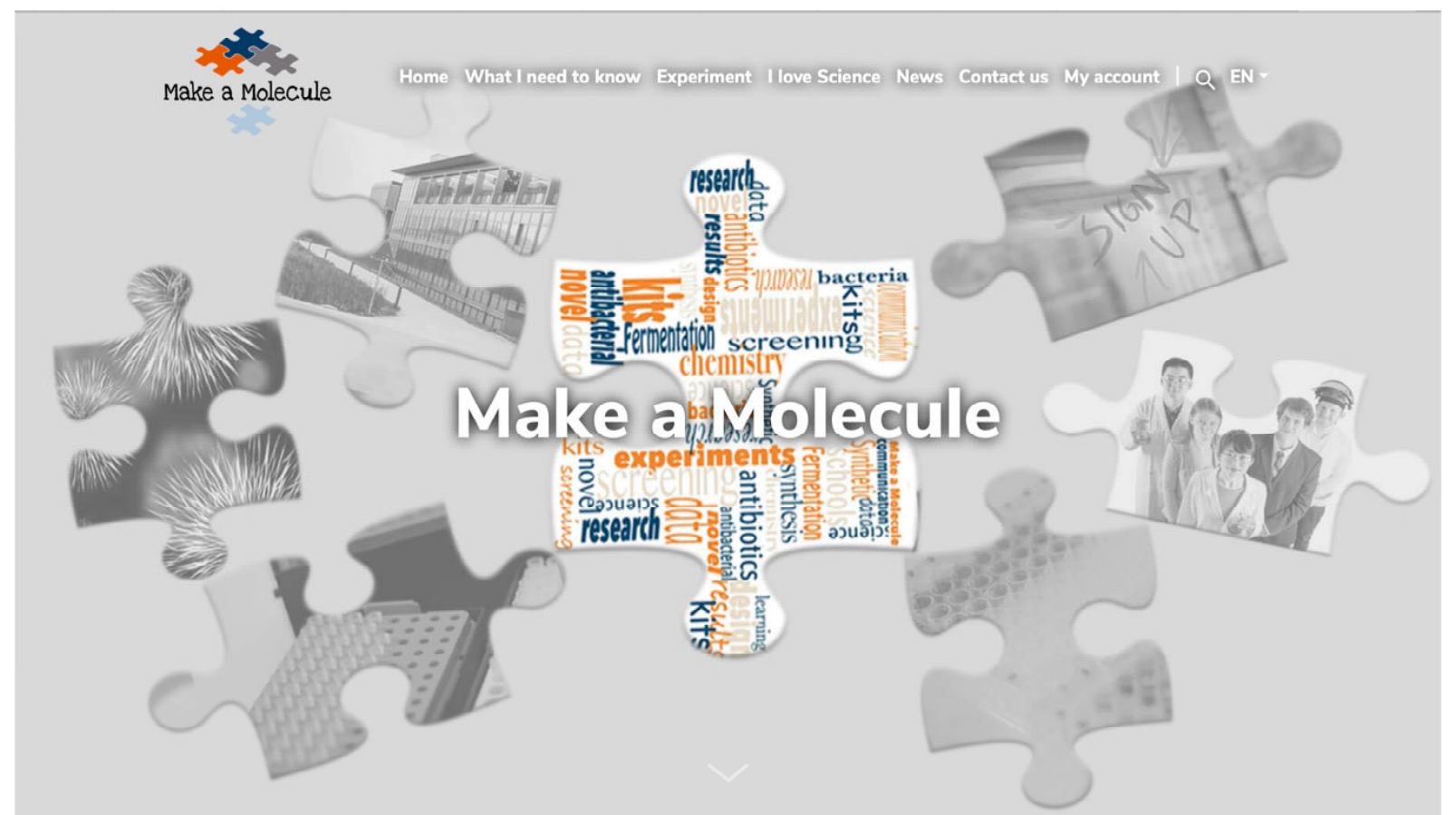

Figure 8. The homepage of our "Make a Molecule" website (http://www.makeamolecule.org), which hosts the experimental guide web application, as well as practical tips videos, a forum section, and background information for students and teachers about chemistry.

Before taking our workshop into schools, the final step was the design of our proposed synthetic fermentation "kits". We decided to provide the small molecule building blocks as pre-weighed solid samples, both to ensure their chemical stability and to allow students to have the opportunity to prepare the required stock solutions prior to the experiment. Our first generation kits also include the necessary chemical and biological equipment to perform the experiment, including pipettes, 96-well plates and assay materials. Larger but still portable equipment, such as a PCR thermal cycler for performing the fermentation reaction and an incubator for culturing bacteria, were loaned and brought to the participating school by an instructor (see Supporting Information for a full list). 


\section{HAZARDS}

We consider synthetic fermentation to be an operationally simple and safe introduction into organic synthesis suitable for a classroom environment. Due to the tiny scale of the chemical reactions performed, the risk of all chemical hazards is reduced accordingly. Nonetheless, hazards remain and appropriate precautions should be taken at all times. All synthetic manipulations should be conducted wearing personal protective equipment (goggles, lab coat, gloves), and all biological processes should be conducted in a sterile environment to avoid contamination. Care should be taken with the flammable $70 \% \mathrm{EtOH}$ solution, particularly in the presence of the sterilizing naked flame. The provided tert-butanol / water (5:1) reaction solvent is an irritant to skin and eyes. Initiators phenylpyruvic acid and 4-hydroxyphenylpyruvic acid are also mild irritants to skin and eyes. Resazurin and tetracycline are mildly hazardous in case of skin or eye contact and hazardous in case of ingestion or inhalation. The synthetic fermentation monomers and terminators have not been tested for safety and should be handled with care.

\section{RESULTS}

Our first Make a Molecule workshops in a classroom environment were conducted in collaboration with faculty and students at Kantonsschule Zurich Nord, with a class of 16 students aged 15-16 years old. It was important to us that our project could function within the boundaries of a typical school timetable. As such, we worked alongside school faculty to devise a class plan that fit within their practical science course schedules (one dedicated science practical session per week, 3.5 hours long) (Figure 9). 


\section{Day 1}

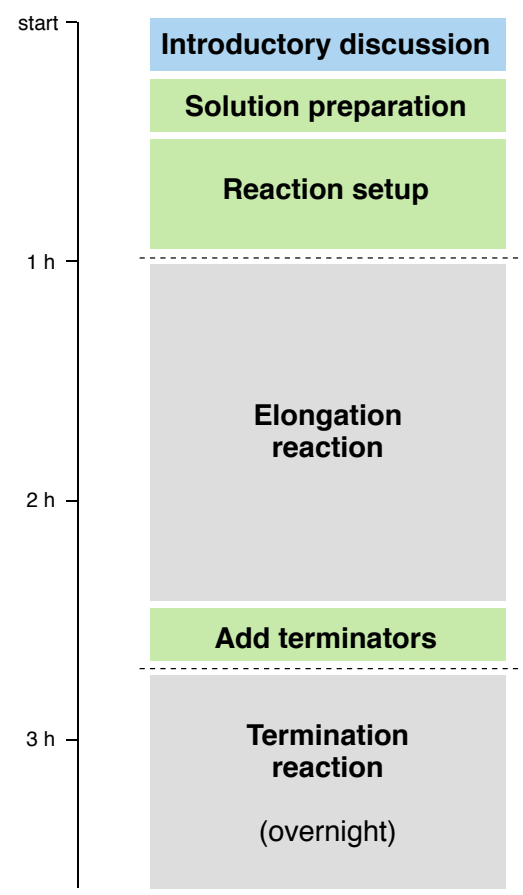

Day 2

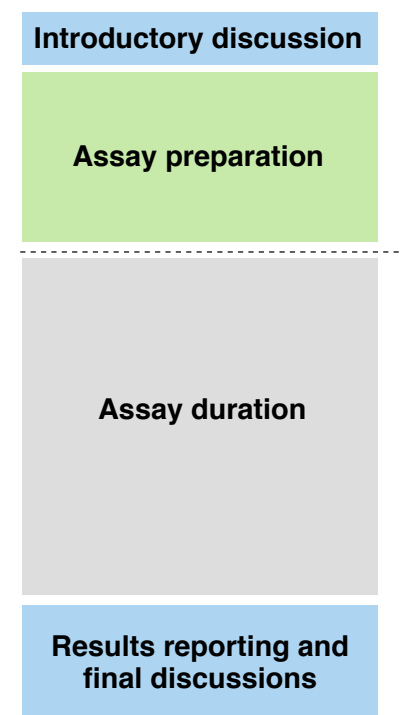

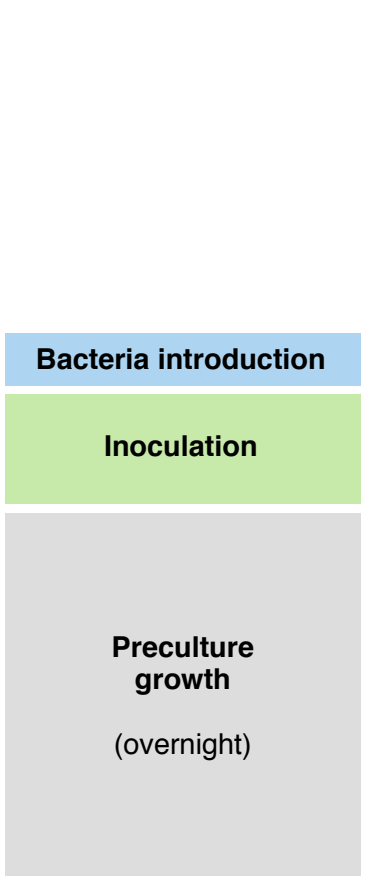

Figure 9. The proposed schedule for initial Make a Molecule workshops. Green boxes indicate experimental work, blue boxes indicate discussions, and grey boxes waiting/processing time. Opportunities for further schedule breaks indicated with dotted lines.

The curriculum we developed involved a short introductory lecture during a theory class time slot, followed by two practical sessions in consecutive weeks. We envisaged that this workshop setup would allow students to follow the drug discovery process from compound synthesis to biological evaluation. A thematic focus on developing and testing a scientific hypothesis (such as "perhaps the choice of initiator is important") would also allow them to understand and practice applying the scientific method, and encourage the students to work as a collective to learn as much as possible about which types of molecules might show antibacterial activity (Figure 10).

The introductory lecture, delivered by a doctoral student from our research group, offered brief insight into the scientific background and concept of the workshop using the materials provided on the Make a Molecule website. Videos from our website containing practical tips for the coming experiments were shown, and medicinal chemistry concepts such as pharmacophores and physicochemical properties were introduced through more familiar ideas like polarity and solubility. As a homework task, the students were each allocated eight reaction wells for their fermentation cultures, and encouraged to design their experiments by choosing their building blocks for each of 
these wells using our web app. During this time, students were also encouraged to directly forward their questions about experimental design to us, through the Technical Support tab on our website.
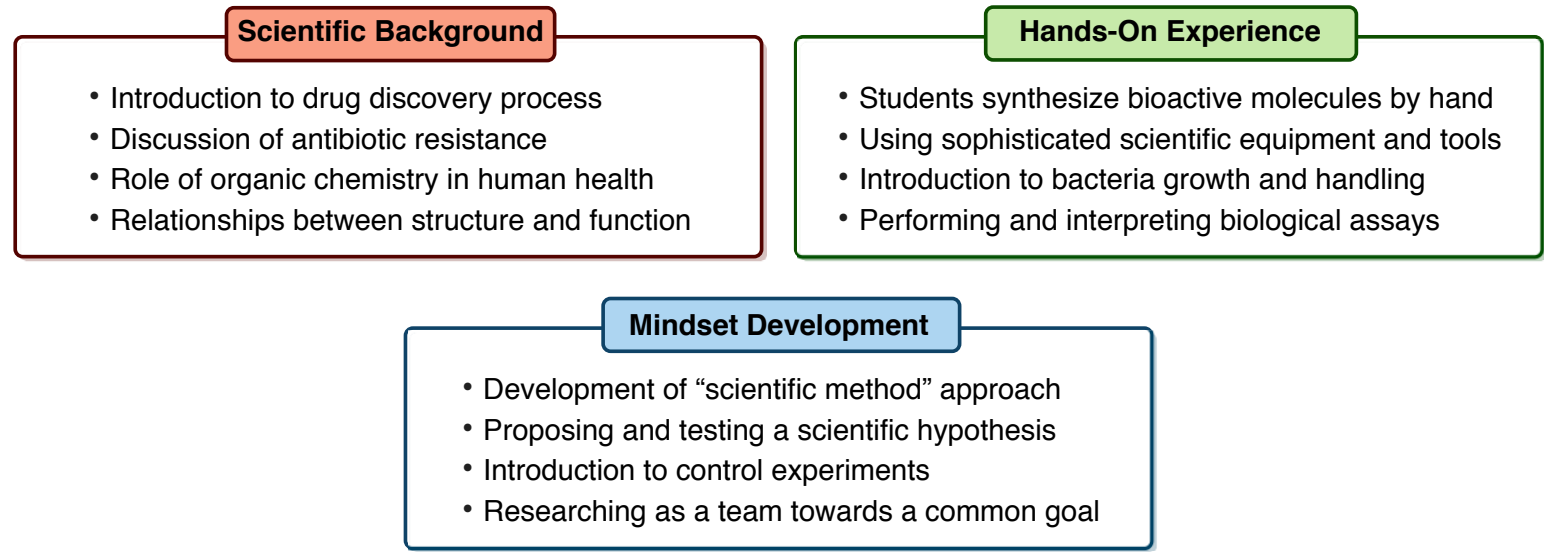

Figure 10. Selected learning outcomes of the Make a Molecule workshops. The workshops hope to provide a scientific background into antibacterial resistance and the drug discovery process and an introduction to scientific ways of thinking, in addition to hands-on experience of chemical synthesis, bacterial handling and biological assays.

In the first three-hour lab session, the students presented their experiment designs, generated the required protocols, and conducted the synthetic fermentation reactions, supervised by a doctoral student. All of the necessary materials and equipment were provided, including the chemical building blocks, which were delivered as pre-weighed samples in sealed vials (Figure 11). To perform the experiment, the students had simply to dissolve the required building blocks in the provided aqueous solvent mixture to generate stock solutions, and pipette their chosen initiators, monomers and terminators into the appropriate wells of a 96-well plate. In our first trial application, this step was among the more time-consuming elements of the experiment. The process was subsequently rendered more efficient by supplying additional pipettes, as well as the use of detached 8-well strips in place of a full 96-well plate, cutting down on the overall waiting time for the students. 


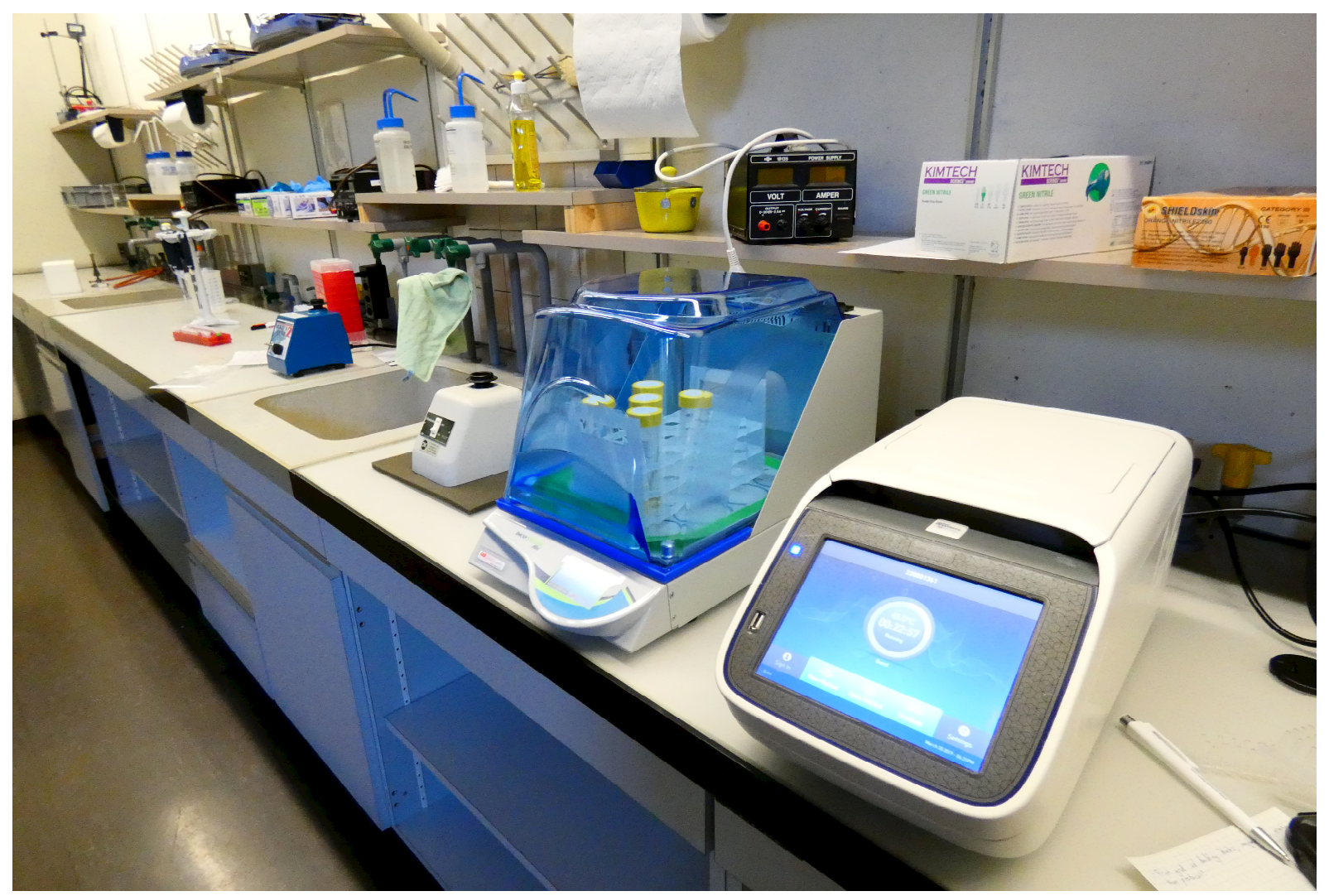

Figure 11. The equipment for our Make a Molecule workshop on a high school laboratory bench top, prepared for a class of students.

During the allotted 1.5 hours for the elongation phase of the synthetic fermentation reaction, the students were taught how to prepare a bacterial preculture and work under a sterile environment. Working in small groups, each sterilized their working surfaces and prepared a bacterial preculture using bacterial growth media and a provided glycerol stock of $B$. subtilis. Previously prepared precultures were shown to the class for comparison, and a short discussion on bacterial growth and assays took place. To fill the final 30 minutes of the reaction time prior to the addition of terminators, an excerpt from a documentary ${ }^{20}$ on antibiotic resistance was shown, and a question and answer session about the topic was hosted. The students then added the appropriate terminators to their fermentation cultures and placed their reactions in the thermal cycler overnight, completing the first session. Once the termination step was complete, the fermentation plate was stored at $4{ }^{\circ} \mathrm{C}$ until the next practical lab session. 


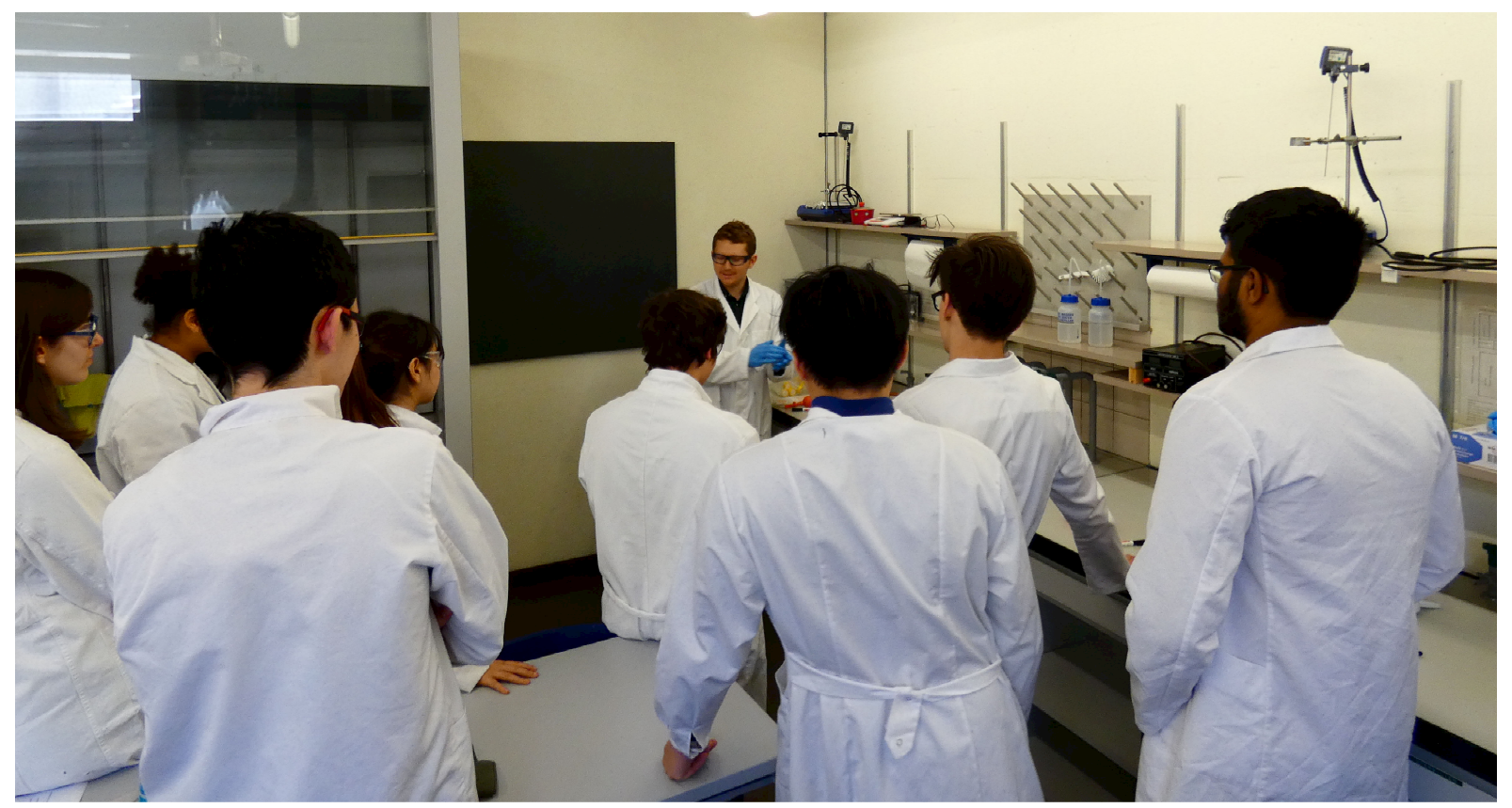

Figure 12. Introductory demonstrations to the first class of students of our Make a Molecule workshop.

In the second three-hour lab session, the students retrieved their fermentation cultures and performed an antibacterial assay. Originally, we planned for the students to culture bacterial samples to the exponential growth phase. However, due to time constraints, the culture of $B$. subtilis used was prepared in advance by a doctoral student. Each student prepared their own colorimetric growth inhibition assay by simply transferring an aliquot of each of their synthetic fermentation wells into the assay plate and adding bacterial stock solution and resazurin dye solution. This was performed in triplicate for all plates, with a $10 \mathrm{mg} / \mathrm{mL}$ tetracycline solution and a solution of PBSdiluted reaction solvent serving as positive and negative controls respectively. This process took approximately 45 minutes for a class of ten students. 


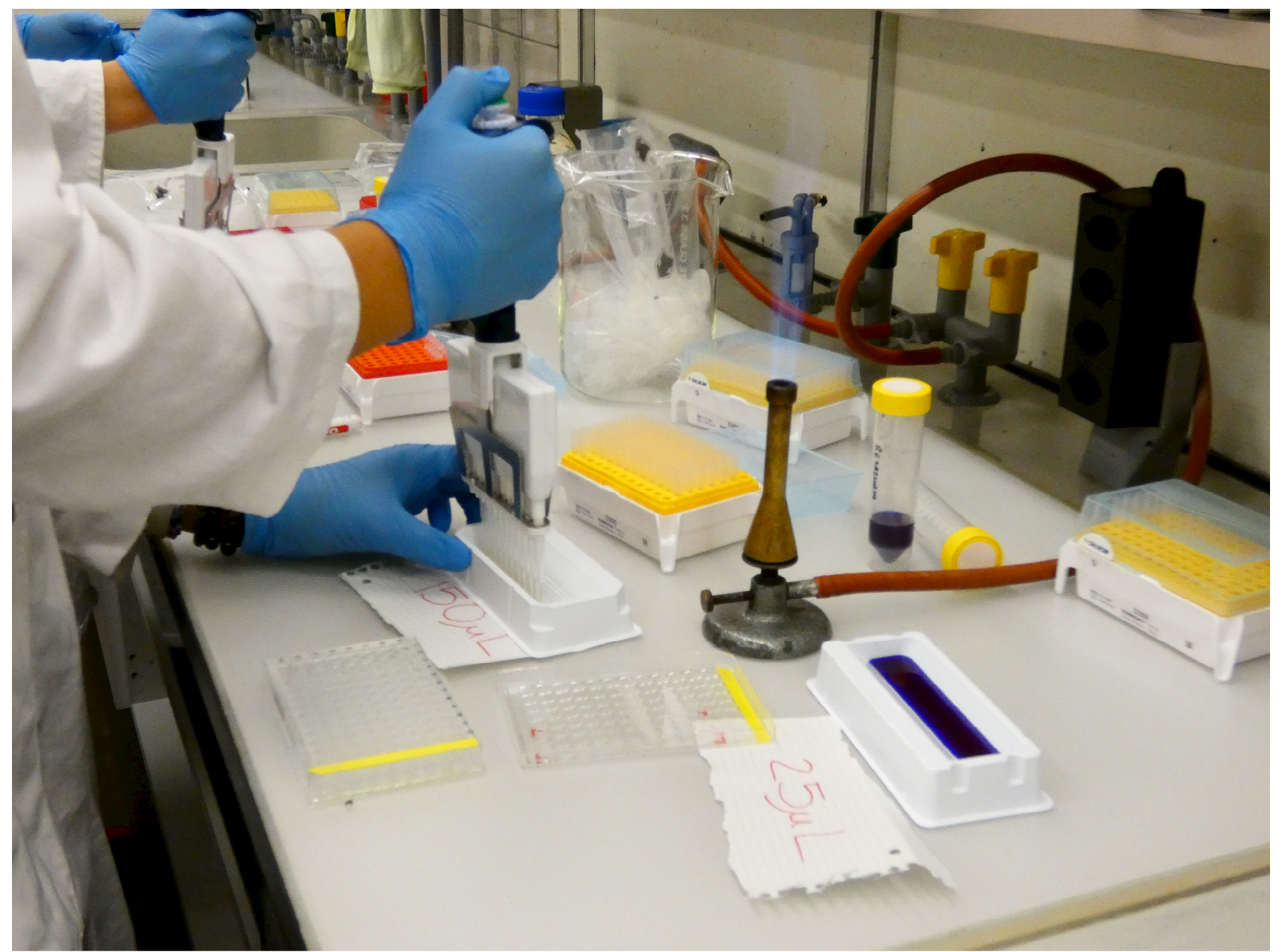

Figure 13. A student prepares the biological assay by combining an aliquot of their synthetic fermentation reaction mixture with a bacterial stock solution and a resazurin dye solution.

The assays were incubated at $37^{\circ} \mathrm{C}$ for 1.5 hours. During the resulting downtime, the remainder of the bacterial resistance documentary was shown to the class. The students were also asked to fill out feedback forms to help us understand which aspects of the program they enjoyed, and which areas required improvement. After 1.5 hours of assay incubation, a clear colorimetric contrast could be seen between the control wells (Figure 14). The results of their assay were recorded by the students, and entered into the web app for compilation. 


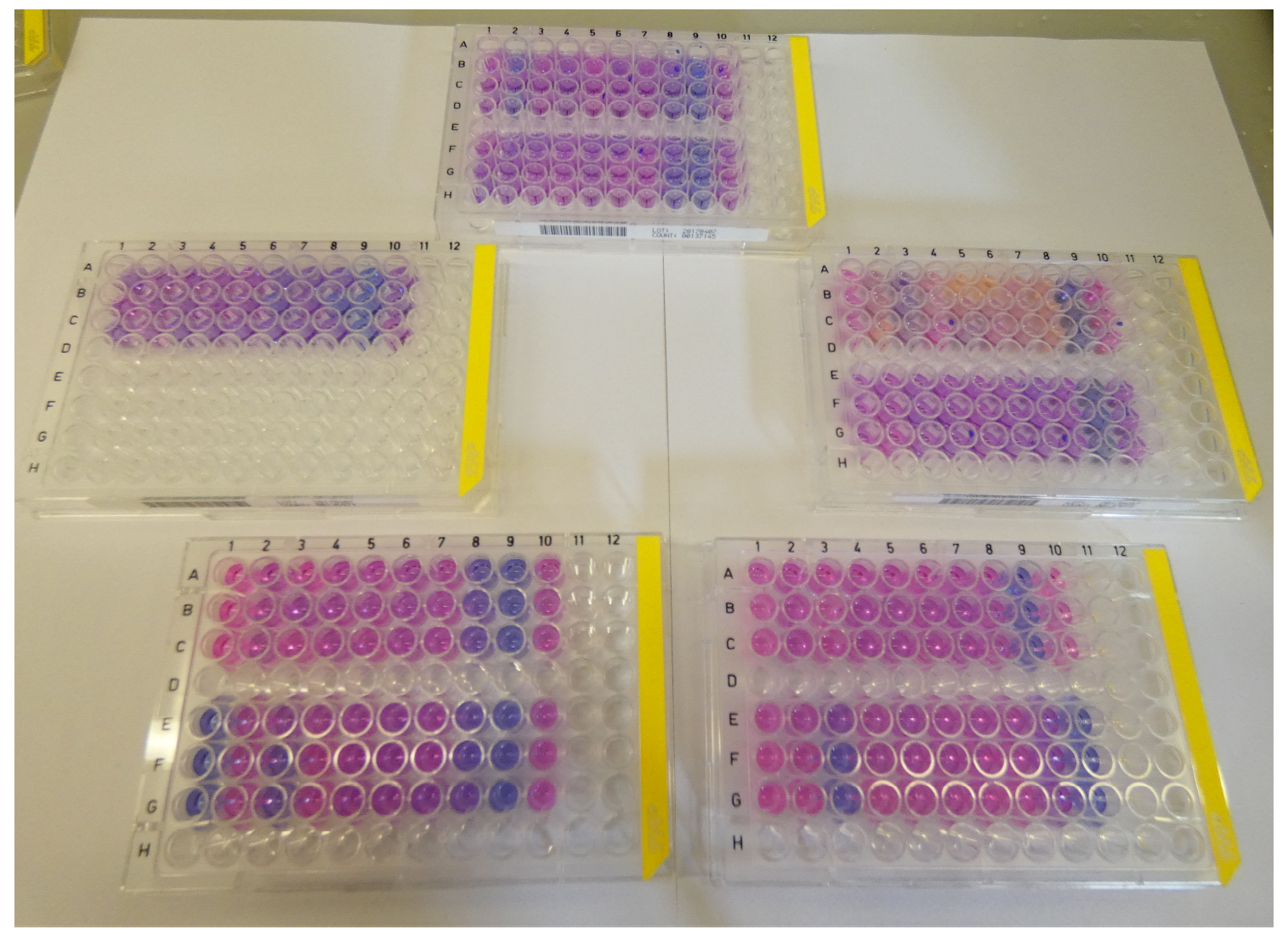

Figure 14. The results obtained from the colorimetric bacterial growth inhibition assay from the first set of Make a Molecule participants. Active wells showing bacterial growth inhibition are blue, while inactive wells are pink. Positive and negative controls are present in column 9 and 10 of each plate.

When designing the workshop, we felt that the challenges of the drug discovery process would be misrepresented if every student was able to discover antibacterial activity on their first attempt. As such, we designed the building block set in the hope that around $25 \%$ of participants would be able to make active molecules. To our satisfaction, four students within the initial class of 16 students were able to make active molecules.

Once the assay was complete and the students had reported their results through our web app, a concluding discussion with the students was initiated. Each student was engaged individually about what they had learned about their initial hypothesis from the obtained results. Even those who had not identified active compounds were able to draw conclusions from their results, and many proposed clear plans for future experiments. For example, one student tested several combinations of initiators and terminators with a single monomer - upon seeing no biological activity, he was able to conclude that this monomer was not a good choice for further tests. Finally, those who identified active wells were encouraged to write the components of their active wells on the laboratory 
blackboard. With these results compiled, the students were able to draw conclusions from their collective work as a research team, identifying trends in these active wells as to which building blocks conferred antibacterial activity, and drawing clear conclusions as a class for which experiments they would like to pursue next.

In this initial trial, doctoral students from our research group acted as external demonstrators for the theoretical and practical aspects of the workshop. With its successful conclusion and the support of the teachers involved, we felt it would be of interest to allow the teachers to conduct the workshop in the absence of external demonstrators to determine to what extent our active participation was necessary. For this iteration of the project, all of the materials were again provided by our team, but the experiments themselves were handled solely by the high school teachers themselves.

These tests proceeded largely successfully, with several students again succeeding in the synthesis and identification of molecules capable of bacterial growth inhibition, and class-wide trends could be clearly observed. Perhaps due to the supervising teachers' lack of hands-on experience with this chemistry, a slightly increased number of mistakes by the students occurred in these workshops. However, we were pleased to see that errors made in the synthetic fermentation step had no adverse effect on the ability of the student to obtain meaningful data, showcasing the robustness of the chemical process. More problematic errors were observed in the assay process, where several students added incorrect volumes of the various assay components (dye solution and/or bacterial stock) to the plate. In one case where an excessive volume of dye solution was added, the color of the resulting assay solution became so saturated that no color change could be seen, even in the positive control well.

However, in spite of these minor complications, the workshops supervised by the teachers proceeded well, and by the end of this initial two-part trial, more than 30 students had taken part in our Make a Molecule program. We believe that, with the help of a short teacher training program, workshops could easily be coordinated by high-school science teachers in a classroom environment without the need for an external demonstrator.

\section{OUTLOOK}


Students and teachers alike responded positively to our Make a Molecule workshop. The teachers remarked upon its complexity and scientific depth in comparison to typical high school chemistry lab exercises, and enjoyed the opportunity to introduce concepts of drug discovery and the role of organic chemistry in human health to the students in an accessible fashion. We were also delighted to see that feedback forms given to the students towards the end of our workshop showed that the vast majority of students were engaged by the experience. Of the critical feedback received, almost all related to easily improved structural factors such as wait times, and after the workshop was complete, more than one student enquired about the possibility to conduct similar work for their diploma project, indicating their enthusiasm for the program.

Since these first trials, we have sought new opportunities to bring our workshop to more students. Kantonsschule Zürich Nord has indicated their enthusiasm for an ongoing relationship with Make a Molecule, suggesting that we may be able to work with a larger student body as part of their "project week" schedule. Working within this framework, we would also hope to expand to other high schools in the Canton of Zürich. We have also made contact with Go Tec!, a Schaffhausen-based educational organization that hosts STEM-themed extracurricular workshops for students, with the aim of partnering with them to bring Make a Molecule to students outside of Zürich for the first time. Through these avenues and others, our goal is to continue the development of Make a Molecule, and establish our unique chemical outreach workshop in high school classrooms across Switzerland and beyond. 


\section{ASSOCIATED CONTENT}

Supporting Information

The Supporting Information is available on the ACS Publications website at DOI: 10.1021/acs.jchemed.XXXXXXX.

\section{AUTHOR INFORMATION}

425

Corresponding Author

*E-mail: bode@org.chem.ethz.ch

\section{ACKNOWLEDGMENTS}

This project is supported by Swiss National Science Foundation AGORA Program Grant \#171667. We would like to thank Dr. Trung Cao and Dr. Yi-Lin Huang for their contributions to the development of synthetic fermentation that led to this program. We thank the faculty and students of Kantonsschule Zürich Nord for their collaboration, particularly Alison Bachofen, Jan Pflug and Sascha Pucillo for aiding in the optimization process. We would also like to thank Applover Software House for web design assistance, and Dr. Vijay Pattabiraman for sourcing building blocks. Image credit for TOC graphic: Haruko Hirukawa.

\section{REFERENCES}

1. TNS BRMB, Public attitudes to Chemistry, Royal Society of Chemistry 2015, available online at: http://www.rsc.org/globalassets/04-campaigning-outreach/campaigning/public-attitudes-to-chemistry/publicattitudes-to-chemistry-research-report.pdf

2. Schummer, J.; Bensaude-Vincent, B.; Van Tiggelen, B. (eds), The Public Image of Chemistry 1-6, 2007 (World Scientific).

3. Hartings M. R.; Fahy, D. Communicating chemistry for public engagement. Nature Chem. 2011, 3, 674677.

4. Lambert, L.; Guiffre, H. Computer science outreach in an elementary school. J. Comput. Sci. Coll. 2009, 24, 118-124. 
5. Benitti, F. Exploring the educational potential of robotics in schools: A systematic review. Comput. Educ. 2012, 58, 978-988.

6. Fitzgerald, M.; Hollow, R.; Rebull, L.; Danaia, L.; McKinnon, D. A Review of High School Level Astronomy Student Research Projects Over the Last Two Decades. Publ. Astron. Soc. Aust. 2014, 31, E037-59.

7. Ross, E. D.; Lee, S. K.; Radebaugh, C. A.; Stargell, L. A. An integrated biochemistry and genetics outreach program designed for elementary school students. Genetics 2012, 190, 305-315.

8. Ting, J. M.; Ricarte, R. G.; Schneiderman, D. K.; Saba, S. A.; Jiang, Y.; Hillmyer, M. A.; Bates, F. S.; Reineke, T. M.; Macosko, C. W.; Lodge, T. P. Polymer Day: Outreach Experiments for High School Students. J. Chem. Ed. 2017 94, 1629-1638.

9. Details available online at: http://www.frankleibfarth.com/outreach/

10. Nowick, J. S.; Brisbois, R. G. The MIT Chemistry Outreach Program: Graduate students presenting chemistry to high school students. J. Chem. Educ. 1989, 66, 668.

11. University of Bristol CHeMneT Program details online at: http://www.chemlabs.bris.ac.uk/outreach/chemnet/Chemnet-regular.html

12. Stepek, I. A.; Bode, J. W. Synthetic fermentation of bioactive molecules. Curr. Opin. Chem. Biol. 2018, 46, 18-24.

13. Huang, Y.-L.; Bode, J. W. Synthetic fermentation of bioactive non-ribosomal peptides without organisms, enzymes or reagents. Nature Chem. 2014, 6, 877-88.

14. Bode, J. W. Chemical Protein Synthesis with the a-Ketoacid-Hydroxylamine Ligation. Acc. Chem. Res. 2017, 50, 2104-2115.

15. Stepek, I. A.; Cao, T.; Koetemann, A.; Shimura, S.; Wollscheid, B.; Bode, J. W. Antibiotic discovery with synthetic fermentation: library assembly, phenotypic screening, and mechanism of action of beta-peptides targeting penicillin-binding proteins. ACS Chem. Biol. 2019, DOI: 10.1021/acschembio.9b00227.

16. SNF AGORA homepage: http://www.snf.ch/en/funding/science-communication/agora/Pages/default.aspx

17. Pflug, J. Maturitätsarbeit Report. Kantonsschule Zürich Nord, 2017.

18. Pucillo, S. Maturitätsarbeit Report. Kantonsschule Zürich Nord, 2017.

19. Bachofen, A. Maturitätsarbeit Report. Kantonsschule Zürich Nord, 2017.

20. Resistance Fighters - Die globale Antibiotikakrise; Broadview TV: Cologne, Germany., 2019. 